Part of Journal of Research of the National Bureau of Standards, Volume 21, November 1938

\title{
CESIUM DISCHARGE UNDER CONDITIONS OF NEARLY COMPLETE IONIZATION
}

\author{
By Fred L. Mohler
}

\begin{abstract}
Measurements of the spectral-intensity distribution of the continuous recombination spectrum have been used to measure electron concentration, $N_{e}$, and electron temperature, $T_{e}$. Using radiation from a $1-\mathrm{mm}$ capillary tube viewed end on, it has been possible to extend measurements to a current density of 300 amperes at pressures up to $17 \mathrm{~mm}$. With increasing current the intensity reaches a flat maximum, and this maximum increases as the square of the pressure. Above 0.3-mm pressure $T_{0}$ is independent of pressure and increases linearly with current. The observations agree quantitatively with the theory that the cesium in the capillary is in temperature equilibrium at a temperature $T_{\mathbf{c}}$ and a pressure equal to the vapor pressure.

Electrical measurements of $T_{\mathrm{e}}$ and $N_{\mathrm{e}}$ have been made in a 5-mm tube with pressures ranging from 0.0045 to $0.3 \mathrm{~mm}$. In this range $T_{\mathrm{s}}$ increases with decreasing pressure. The gas temperature is not in equilibrium with $T_{e}$, and $N_{e}$ has a value which is higher than that corresponding to temperature equilibrium.
\end{abstract}

\section{CONTENTS}

Introduction . . . . . .

II. Methods_...

III. Procedure

IV. Spectroscopic results

V. Interpretation of spectra $\ldots \ldots$

VI. Electrical measurements . . .

VII. Discussion

\section{INTRODUCTION}

In an electric discharge there is commonly what is termed a plasma. The positive ions, because of their low mobility, produce a positive space charge which holds the electrons in the space, and the electric field resulting from the space charge is limited to a thin sheath over the walls of the tube. In the space there is an electron gas mixed with the atomic gas and by means of electrical measurements with probe wires, the energy distribution and concentration of the electrons can be measured. This energy distribution is commonly a Maxwell distribution and is conveniently expressed as a temperature. The definition of electron temperature is based solely on the kinetic theory conception of temperature, with no implication as to the existence of equilibrium between atoms and electrons. 
A series of papers ${ }^{1}$ on the interactions of electrons and atoms in the cesium discharge has shown that with increasing pressure and current density there is an approach toward equilibrium between the state of excitation and ionization of the gas and the electron temperature. With current densities of the order of an ampere and pressures over $0.005 \mathrm{~mm}$ the number of atoms in the first excited state is nearly equal to the equilibrium value for a temperature equal to the electron temperature. With pressures less than $0.3 \mathrm{~mm}$ the population of higher excited states and ionized states is always less than the equilibrium value.

Two technical limitations make it impossible to apply the conventional methods with higher pressures and currents. First, the use of probe wires to measure electron concentrations is impossible with high currents and high pressures. Second, the vapor pressure, but not the vapor density, is known in the cesium experiments, and in an intense discharge there is no basis for estimating the gas temperature and concentration of neutral atoms.

Several types of experiments, notably the experiments of Elenbaas ${ }^{2}$ with the mercury arc, indicate that at pressures of the order of an atmosphere the gas temperature is equal to the electron temperature. However, recent spectroscopic studies by Barnes and Adams ${ }^{3}$ indicate that considerations of spectral intensity, intensity distribution, and heat loss lead to three quite different figures for the temperature. These considerations make it of interest to extend the study of the cesium discharge to higher pressures and currents, and this paper describes experiments with methods designed to avoid, as far as possible, the limitations of more conventional methods.

\section{METHODS}

It has been shown ${ }^{4}$ that measurements of the intensity distribution in the continuous recombination spectrum give a basis for measuring electron temperature and concentration, and the method should entirely avoid the limitations of probe measurements. The uncertainty as to the concentration of atoms can at least be minimized if conditions of nearly complete ionization can be attained, for then the number of neutral atoms per cubic centimeter becomes of trivial importance for most considerations.

The continuous recombination spectrum consists of bands extending from each series limit to higher frequencies and the radiation at a frequency interval, $\nu-\nu_{0}$, beyond the limit comes from electrons with a kinetic energy corresponding to this frequency difference

$$
h\left(\nu-v_{0}\right)=\frac{1}{2} m v^{2}=E .
$$

Thus, the intensity distribution in the band depends on the energy distribution, or temperature of the electrons. For the case of the band beyond the subordinate series limit, $6 \mathrm{P}$ of cesium, the relation

1 F. L. Mohler, Power input and dissipation in the positive column of a caesium discharge, BS J. Research 9, 25 (1932) RP455; Collisions of the first and second kind in the positive column of a caesium discharge, BS J. Research 9, 493 (1932) RP485; Reversal temperature and population of excited states in the cesium discharge, J. Research NBS 16, 227 (1936) RP869; Electron concentration and spectral-intensity distribution in a cesium discharge, J. Research NBS 17, 849 (1936) RP948.

2 W. Elenbaas, Physica 1, 211, 673 (1934); 2, 155, 169 (1935).

${ }^{3}$ Emission of radiation in the positive column of a mercury arc by B. T. Barnes and E. Q. Adams, Phys. Rev. 53, 545 (1938). The mechanism of the positive column in mercury vapor at intermediate pressures by E. Q. Adams and B. T. Barnes, Phys. Rev. 53, 557 (1938).

4 Mohler, J. Research NBS 17, 849 (1936) RP948. 
between the intensity, $J_{\lambda}$, the electron concentration, $N_{e}$, and the electron temperature, $T_{e}$, have been determined by experiment. ${ }^{5}$ A convenient form of the relation for the present purpose is

$$
\lambda J_{\lambda}=0.516 \times 10^{-18} \frac{N_{e}^{2}}{T_{e}^{3 / 2}} e^{-E / k} T_{e},
$$

where $J_{\lambda}$ is the radiation flux, in ergs, in all directions in unit range of wave length ( $\lambda J$ is independent of the unit). It follows that a plot of $\log \lambda J$ versus $E$ gives a line of slope inversely proportional to $T_{e}$, while the intensity at the limit $(\lambda=0.5 \mu)$ is ${ }^{6}$

$$
J_{\lambda}=1.03 \times 10^{-18} N_{e}^{2} / T_{e}^{3 / 2} .
$$

At pressures less than $0.3 \mathrm{~mm}$ it has been possible to use an electrical method of measuring $T_{e}$ and $N_{e}$. The random electron-current density was too high to measure with a positive probe, but it was possible to measure the positive current to a probe flush with the wall, and also the electron current reaching this electrode at potentials a volt or more negative with respect to the space potential. The electron temperature was obtained from these measurements by the usual semilogarithmic current-voltage curves. The electron concentration at the axis of the tube is related to the positive-ion current density by the "ion-current equation".?

$$
N_{e}=2.08 \times 10^{16} I_{+} T_{e}^{-1 / 2} .
$$

This equation has been derived for the case of low pressure and long mean free paths, but experience shows that it remains approximately true at fairly high pressures.

\section{PROCEDURE}

In order to obtain high current densities without the use of excessive currents, a section of narrow tubing between two wider tubes has been used. Figure 1 illustrates the type of tube used for the spectroscopic measurement of $N_{e}$ and $T_{e}$. A $1-\mathrm{mm}$ capillary $1 \mathrm{~cm}$ long was commonly used, and for extreme conditions, the capillary was of quartz with quartz Pyrex seals connecting it to the rest of the tube. The discharge was viewed end-on through a thin, blown Pyrex window. The quartz is badly attacked by the cesium, but the Pyrex window was only slightly discolored with

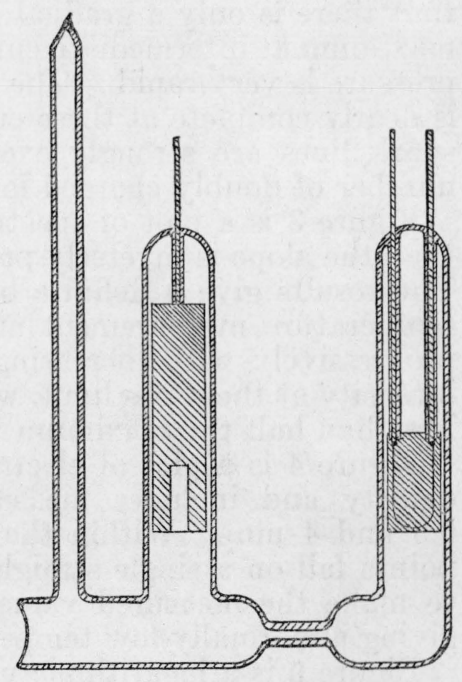

Figure 1.-Type of tube used for spectroscopic measurement of $N_{e}$ and $T_{e}$. vapor pressures as high as $1 \mathrm{~cm}$. The reaction with quartz quickly uses up all of the cesium at pressures above a few millimeters, and exposures had to be planned in advance and made very quickly. Intensities have been measured by densitometer comparison with exposures to a calibrated strip lamp.

${ }^{3}$ Mohler, BS J. Research 10, 771 (1933) RP565.

6 The $5 \mathrm{D}$ continuum overlaps the $6 \mathrm{P}$ limit and contributes to the intensity at wave lengths less than $5000 \mathrm{~A}$ by an amount which depends on $T_{e}$ but is of the order of 30 percent.

7 Tonks and Langmuir, Phys. Rev. 34,876 (1929). 
There are many factors of uncertainty in this work. There is necessarily a pressure gradient with a high current density, ${ }^{8}$ but appearances indicate that this is comparatively small in the absence of foreign gas. Evolution of gas can reduce or extinguish the discharge though the discharge tends to clean up the evolved gas and resume normal operation. The slight discoloration of the glass can be a serious source of error in measuring $T_{e}$, and radiation in the line of sight outside the capillary influences the intensity measurements. The time factor has made it impractical to use some obvious precautions to reduce these uncertainties.

Electrical measurements were made in a $5-\mathrm{mm}$ tube with probe surfaces flush with the tube wall. These surfaces were the ends of copper wires $2 \mathrm{~mm}$ in diameter, insulated except for the ends with close-fitting glass tubes. The small exposed area and large radiating area prevented thermionic emission from overheating. The 5-mm glass tube could be operated at currents up to $20 \mathrm{amp} / \mathrm{cm}^{2}$.

At low pressures there is a sharply defined upper limit to the current which can be carried without violent oscillations in current and voltage,${ }^{9}$ though other factors commonly limited the current range in this work.

\section{SPECTROSCOPIC RESULTS}

Figure 2 shows a series of 10 -sec. exposures at a pressure of $0.3 \mathrm{~mm}$ and of 0.1 -sec exposures at 4 -mm pressure. The striking feature is that there is only a gradual change in intensity with current, with a maximum at intermediate currents, while the change in intensity with pressure is very rapid. The phenomena suggest that single ionization is nearly complete at these currents. At $0.33 \mathrm{~mm}$ and $150 \mathrm{amp}$ the spark lines are strongly excited, but probably even in this case the number of doubly charged ions is negligible.

Figure 3 is a plot of spectral-intensity distribution on a scale such that the slope is inversely proportional to the temperature (see eq 2). The results give a definite but not very accurate basis for electrontemperature measurement and show that the temperature increases progressively with increasing current. Ordinates at $E=0$ give the intensity at the series limit, which is a maximum near $75 \mathrm{amp} / \mathrm{cm}^{2}$ and less than half the maximum value at $150 \mathrm{amp} / \mathrm{cm}^{2}$.

Figure 4 is a plot of electron temperature as a function of current density and includes measurements at various pressures between 0.3 and $4 \mathrm{~mm}$. Within the range of experimental uncertainty, the points fall on a single straight line. Discoloration of the glass tends to make the measured values low and some series of measurements giving abnormally low temperatures have been discarded.

Figure 5 is a logarithmic plot of intensity near the series limit as a function of pressure. The points are the intensity for a current giving maximum intensity, except for the two highest points which were determined at a single current of $300 \mathrm{amp} / \mathrm{cm}^{2}$. The slope of the line indicates that the intensity varies nearly as the square of the pressure. The current of maximum intensity increases with the pressure, so a plot of intensity at constant current would give a curve steeper than this at low pressures and of gradually decreasing slope.

${ }^{8}$ Langmuir, J. Franklin Inst. 196, 751 (1923).

Tonks, Theory and phenomena of high current densities in low-pressure arcs. Trans. Electrochem. Soc. 72, 167 (1937). 


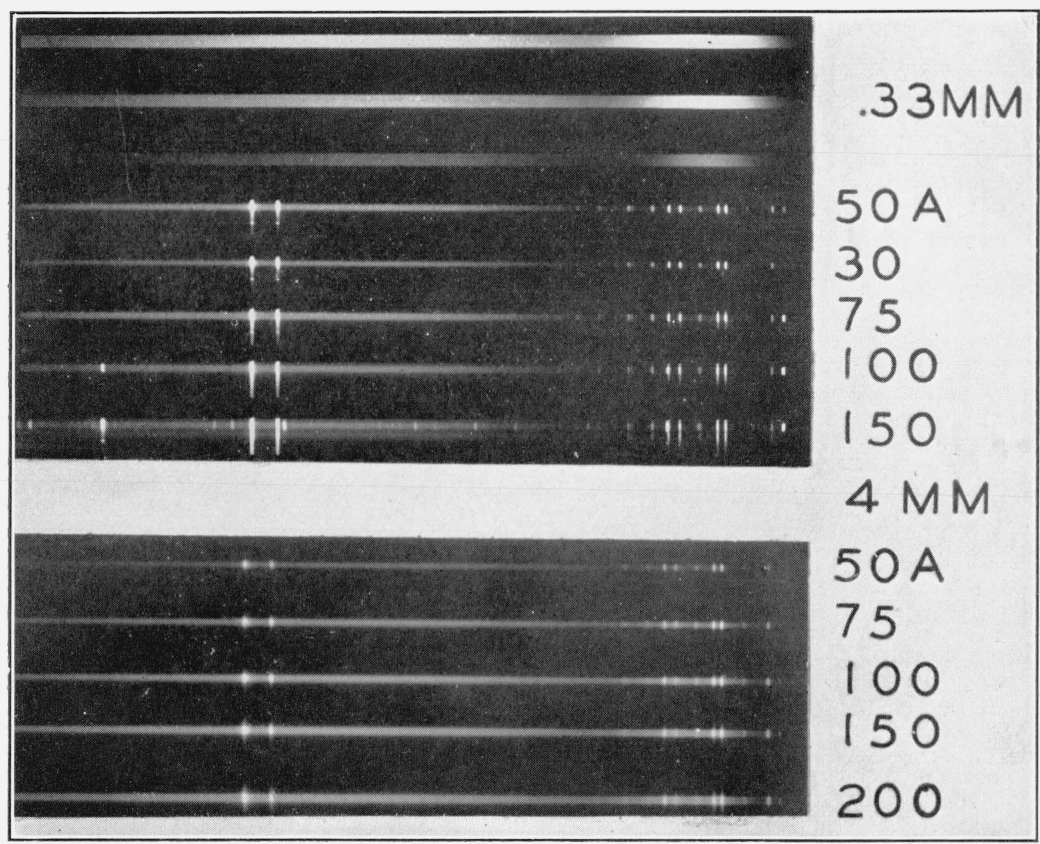

Figure 2.- Spectra of cesium discharge in 1-mm capillary in the range 4000 to 6000 A, left to right.

The upper group are 10 -sec exposures to strip lamp at $1,665^{\circ} \mathrm{K}$ and to a discharge at 0.33 -mm pressure. The lower group are 0.1 -sec exposures at 4-mm pressure. 


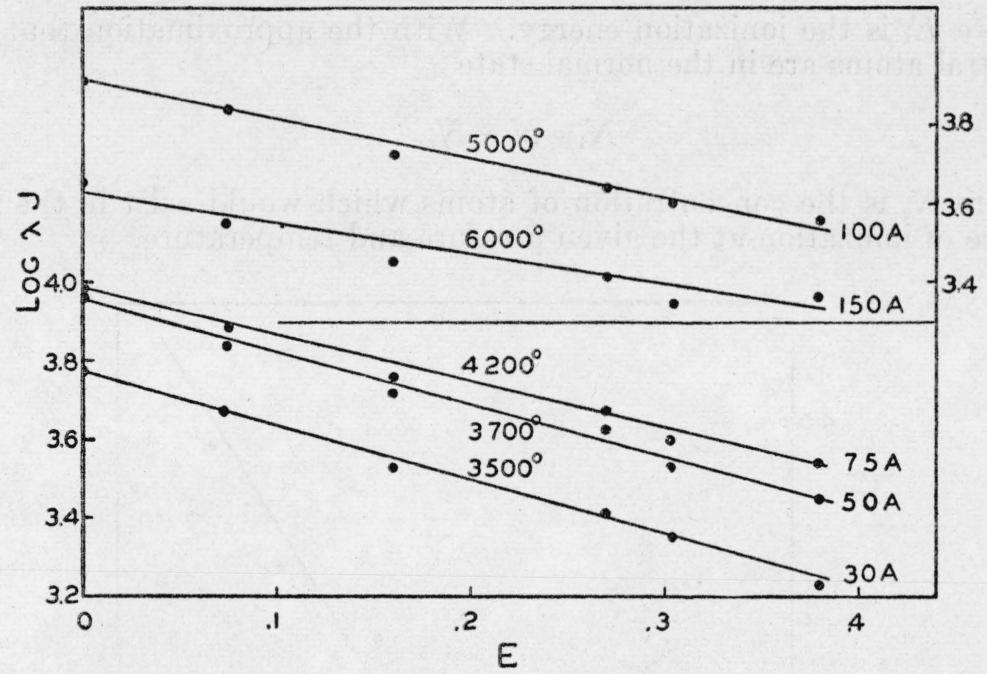

FIGURE 3.-Intensity distribution in the cesium continuous spectrum between 5000 and $4300 \mathrm{~A}$ at a pressure of $0.6 \mathrm{~mm}$, plotted on a scale to give electron temperature, (see eq 2).

\section{INTERPRETATION OF SPECTRA}

It is of interest to compare these observations with the intensity of radiation which would be emitted by cesium vapor in thermal equilib-

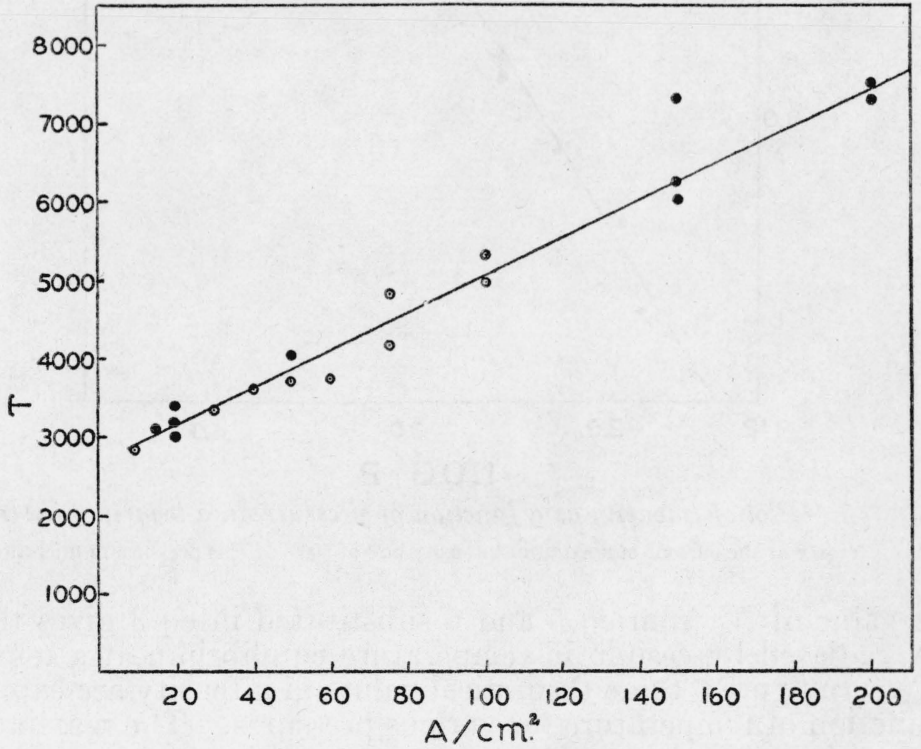

FIGURE 4.-Electron temperature as a function of current density. Vapor pressures between 0.3 and $4 \mathrm{~mm}$.

rium at a given temperature and pressure. The concentration of electrons or ions $\left(N_{e}=N_{+}\right)$relative to the concentration of normal atoms $N_{1}$ is by Saha's equation

$$
\frac{N_{e}^{2}}{N_{1}}=2.43 \times 10^{15} T^{3 / 2} e^{-E \sharp / k T},
$$


where $E_{i}$ is the ionization energy. With the approximation that all neutral atoms are in the normal state

$$
N_{1}=N_{0}-N_{e},
$$

where $N_{0}$ is the concentration of atoms which would exist in the absence of ionization at the given pressure and temperature.

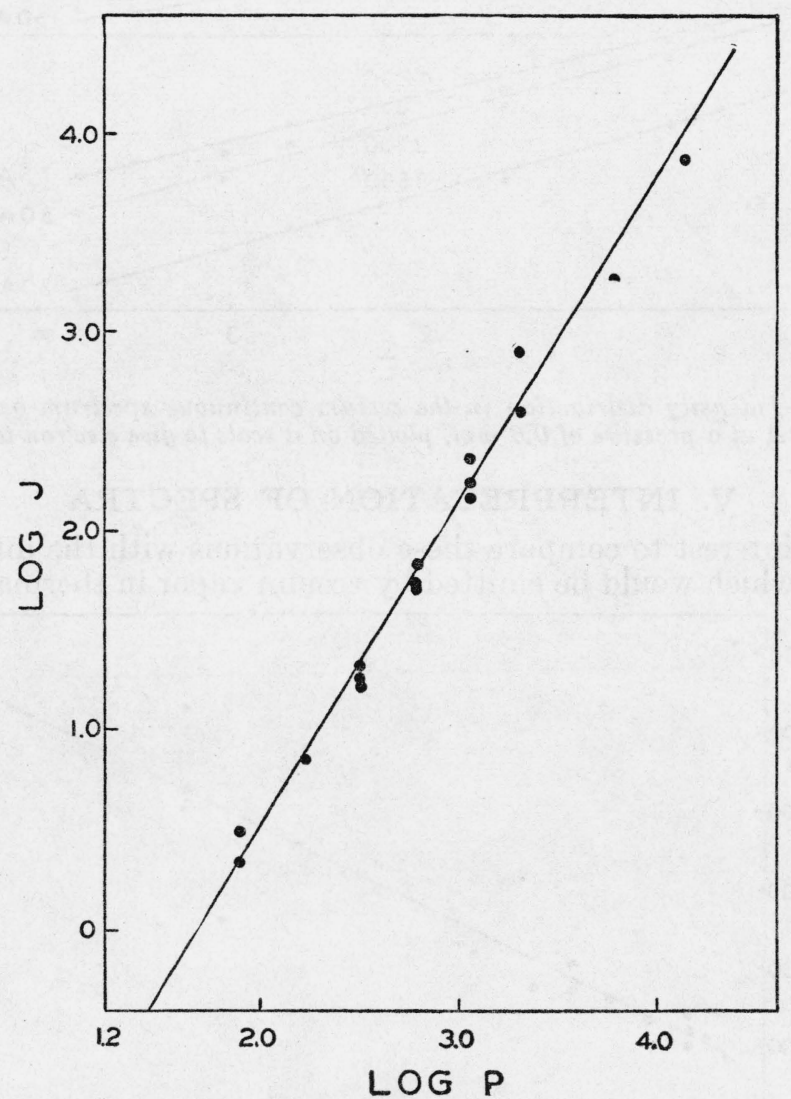

FIGURE 5.-Plot of intensity as a function of pressure on a logarithmic scale.

$J$ is intensity at the current of maximum intensity and at $5000 \mathrm{~A} ; P$ is pressure in microns.

The value of $N_{e}$ from eq 5 and 6 substituted in eq 3 gives the intensity radiated by cesium in temperature equilibrium at a temperature $\mathrm{T}_{e}$. In figure 6 these theoretical values of intensity are expressed as a function of temperature for various pressures. The maximum of each curve is attained when about 90 percent of the atoms are ionized. With a further increase in temperature the decrease in vapor density and in the recombination coefficient more than compensates for the greater percentage of ions. At the highest temperatures plotted the ionization is over 99 percent complete.

In figure 7 the points are observed values of $\log J$ as a function of current density at different pressures. The curves are theoretical values of $\log \bar{J}$ for equilibrium at a temperature equal to the electron temperature. That is, they are the curves of figure 6 expressed in 


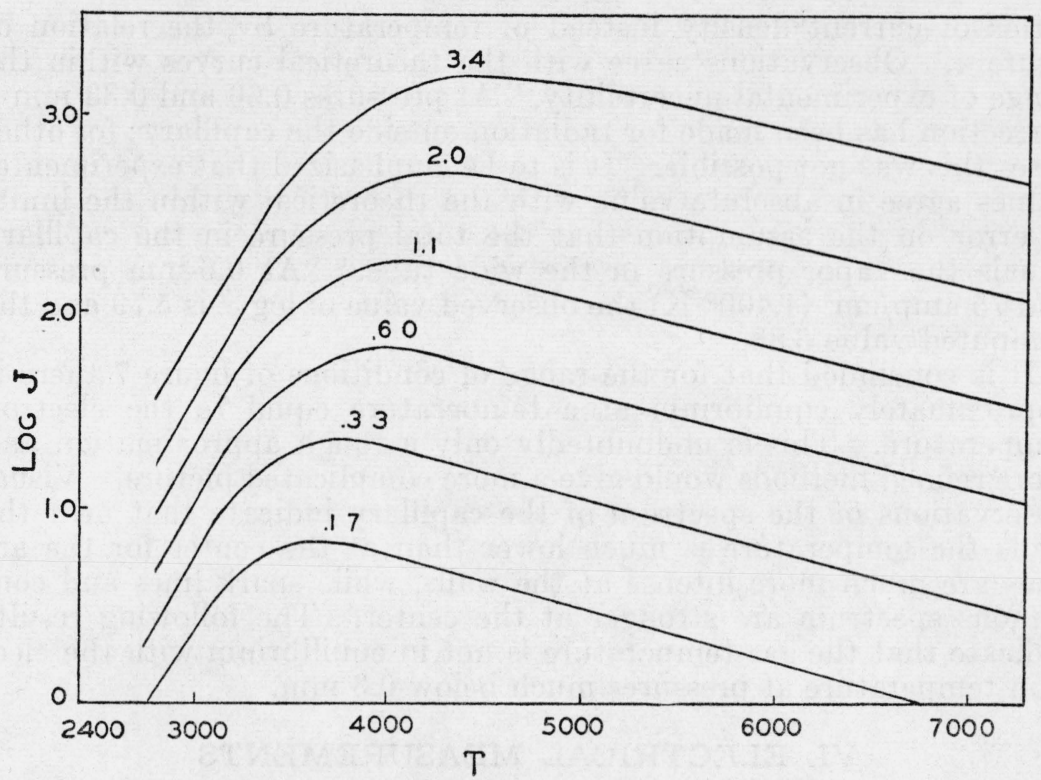

FIGURE 6.-Theoretical curves of $\log J$ as a function of temperature for temperature equilibrium for pressures $(\mathrm{mm})$ indicated on curves.

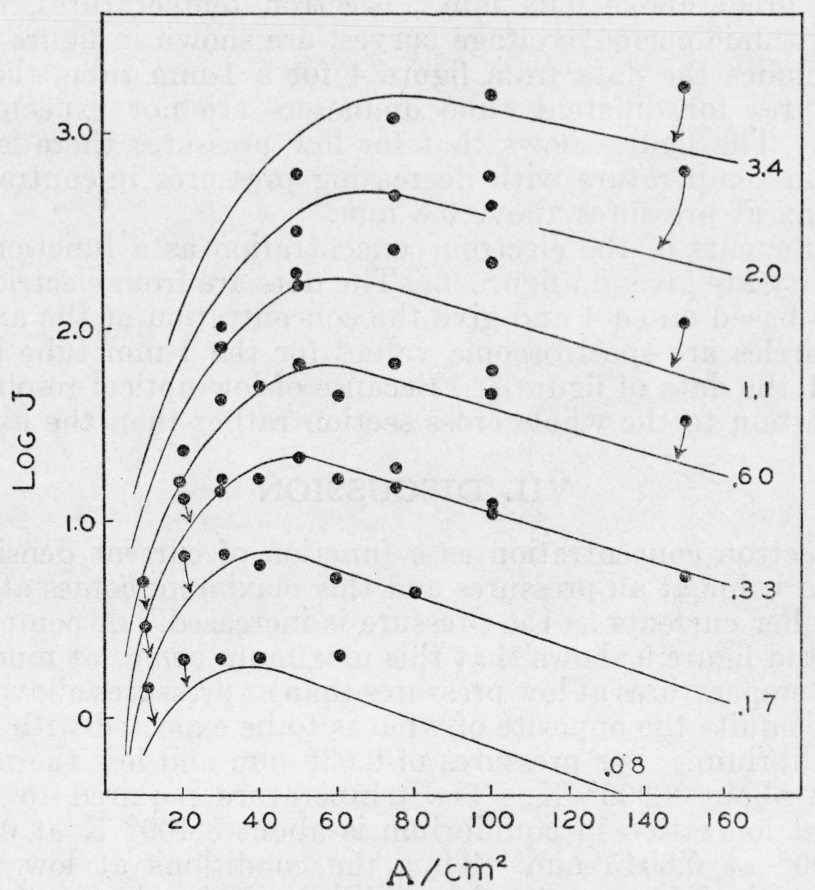

Figure 7.-Points are the measured values of $\log J$ as a function of current density, while the curves are theoretical values for temperatures given by figure 4. 
terms of current density instead of temperature by the relation of figure 4. Observations agree with the theoretical curves within the range of experimental uncertainty. At pressures 0.60 and $0.33 \mathrm{~mm}$ a correction has been made for radiation outside the capillary; for other cases this was not possible. It is to be emphasized that experimental values agree in absolute value with the theoretical within the limits of error on the assumption that the total pressure in the capillary equals the vapor pressure in the wide tube..$^{10}$ At $0.6-\mathrm{mm}$ pressure and $75 \mathrm{amp} / \mathrm{cm}^{2}\left(4,400^{\circ} \mathrm{K}\right)$ the observed value of $\log J$ is 5.79 and the computed value 5.88 .

It is concluded that for the range of conditions of figure 7 there is approximately equilibrium at a temperature equal to the electron temperature. This is undoubtedly only a rough approximation and more refined methods would give a more complicated picture. Visual observations of the spectrum of the capillary indicate that near the walls the temperature is much lower than at the center for the arc lines are much more intense at the walls, while spark lines and continuous spectrum are stronger at the center. The following results indicate that the gas temperature is not in equilibrium with the electron temperature at pressures much below $0.3 \mathrm{~mm}$.

\section{ELECTRICAL MEASUREMENTS}

Electrical measurements in a 5 -mm tube have been made in the pressure range $0.0045 \mathrm{~mm}$ to $0.3 \mathrm{~mm}$, though the experimental uncertainty is great above $0.08 \mathrm{~mm}$. Electron temperatures, based on semilogarithmic current voltage curves, are shown in figure 8 . This figure includes the data from figure 4 for a $1-\mathrm{mm}$ tube, though the temperatures for different tube diameters are not expected to be identical. The figure shows that for low pressures there is a rapid increase in temperature with decreasing pressures in contrast to the phenomena at pressures above $0.3 \mathrm{~mm}$.

Measurements of the electron concentration as a function of current density are given in figure 9 . The dots are from electrical measurements based on eq 4 and give the concentration at the axis of the tube. Circles are spectroscopic values for the 1-mm tube based on eq 3 and the data of figure 7. Because of low optical resolution the values pertain to the whole cross section rather than the axis of the tube.

\section{DISCUSSION}

The electron concentration as a function of current density has a maximum value at all pressures and this maximum comes at progressively higher currents as the pressure is increased. A comparison of figure 8 and figure 9 shows that this maximum comes at much higher electron temperatures at low pressures than at pressures above $0.3 \mathrm{~mm}$, and this is quite the opposite of what is to be expected with temperature equilibrium. For pressures of $0.039 \mathrm{~mm}$ and less the maximum comes at about $8,000^{\circ} \mathrm{K}$. The temperature required to maintain 90 -percent ionization in equilibrium is about $3,400^{\circ} \mathrm{K}$ at $0.039 \mathrm{~mm}$ and $3,000^{\circ}$ at $0.0045 \mathrm{~mm}$. Thus the conditions at low pressures depart greatly from a state of equilibrium. The electron concentration is higher than would exist in equilibrium at the same temperature.

10 For complete ionization $N$, then equals $1 / 2 N_{0}$ and not $N_{0}$. Curves of figure 6 have not been recomputed but simply changed by a constant factor in figure 7 , so they are slightly in error at low currents. 
At $0.0045 \mathrm{~mm}$ the observed maximum value of $N_{e}$ is $1.25 \times 10^{13}$, while there would be $0.3 \times 10^{13}$ in equilibrium at $8,000^{\circ}$.

This discrepancy between conditions in a low-pressure discharge as compared with a state of temperature equilibrium at a temperature $T_{e}$ is entirely in accord with conventional views as to the mechanism of a low-pressure discharge. At low pressures ions recombine on the walls of the tube and evaporate as neutral atoms at a temperature com-

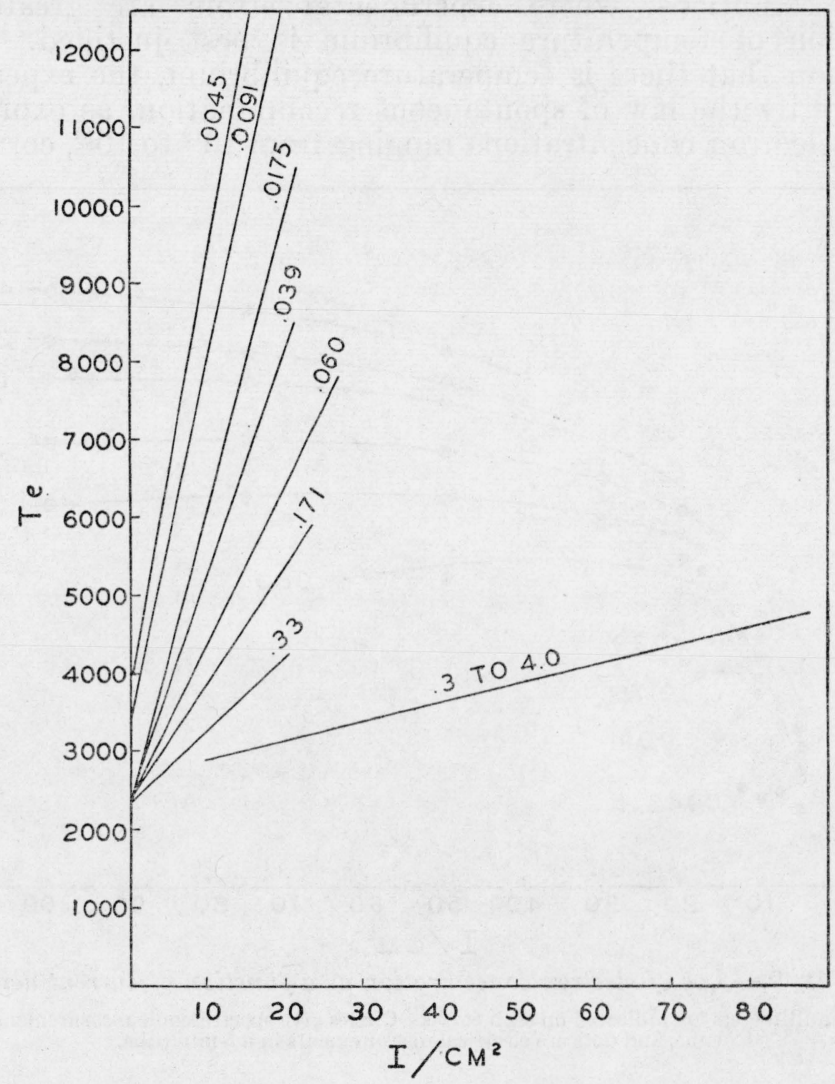

FIgURE 8.-Electron temperature versus current densities in a 5-mm tube.

Numbers on the curves are vapor pressures in millimeters. The lower curve gives the data of figure 4 for a $1-\mathrm{mm}$ tube.

parable with the wall temperature. This keeps both the gas temperature and the ion temperature far below the electron temperature, for the momentum given to the ion in ionization by collision is negligible.

The significant new result of this work is that in an intense discharge at pressures as low as $0.3 \mathrm{~mm}$ the gas temperature has become nearly equal to $T_{e}$. Probably volume recombination has at these pressures become large in comparison with wall recombination and this combined with the short mean free path of electrons in a highly ionized gas makes it possible to maintain a gas temperature far in excess of the wall temperature. The approach to equilibrium between gas temperature and $T_{e}$ is in the pressure range 0.08 to $0.3 \mathrm{~mm}$. Above $0.3 \mathrm{~mm}$ it is safe to assume that the percentage of ionization and the concentration of neutral atoms $N_{1}$ are approximately what would be 
computed for equilibrium at a temperature $T_{e}$. Thus a measurement of any two of the quantities, pressure, $N_{e}$, and $T_{e}$, determines the conditions in the discharge. At lower pressures there is no basis for estimating $N_{1}$ and the percentage ionization.

Theoretical values of the maximum number of ions which can exist in temperature equilibrium at a given pressure furnish a valuable check on experimental methods, and it is very fortunate that with extreme conditions, where experimental errors are greatest, the assumption of temperature equilibrium is best justified. On the assumption that there is temperature equilibrium, the experimental results verify the law of spontaneous recombination, as expressed in eq 2 for electron concentrations ranging from $10^{12}$ to $10^{16}$, correspond-

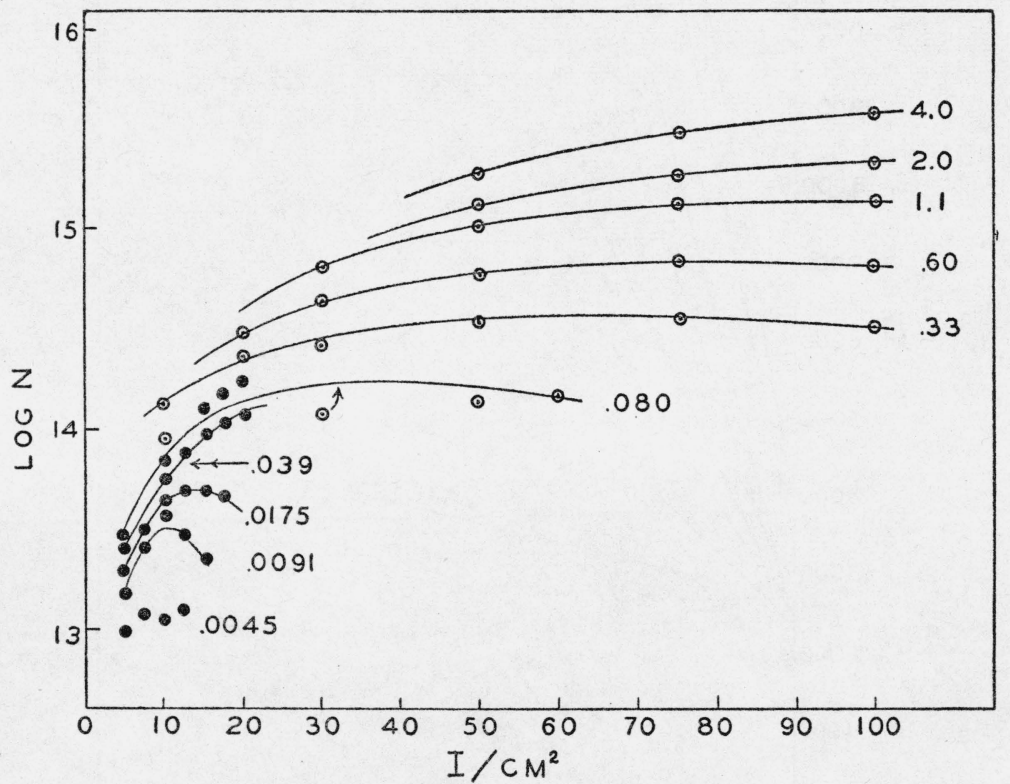

FIGURE 9.-Log of electron concentration as a function of current density.

Pressures in millimeters are indicated on each curve. Circles give spectroscopic measurements in a 1-mm tube, and dots are electrical measurements in a $5-\mathrm{mm}$ tube.

ing to a range of $10^{8}$ in intensity. ${ }^{11}$ This conclusion is consistent with the postulate of radiation theory that transition probabilities are purely atomic properties independent of pressure, temperature, etc., for the coefficient of eq 2 is a transition probability. A recent paper of Bartels ${ }^{12}$ proposes a theory that definitely rejects conventional radiation theory on the grounds that experiments on "Atomgrenz-continua" necessitate such a step. The cesium experiments (the only quantitative experiments) give no support to this viewpoint.

The data presented here on the electron concentration and temperature in discharges of high current density will be used in subsequent papers on the conductivity of an ionized gas and on the number of quantized states that can exist in an ionized gas.

Washington, August 9, 1938.

${ }_{11}$ This range includes published results with low pressures and currents. J. Research NBS 17, 849 (1936) RP948.

${ }_{12}$ Bartels, Z. Physik 105, 704 (1937). 